\title{
Extreme intralist similarity in serial learning
}

\author{
M. RAY DENNY, MICHIGAN STATE UNIVERSITY \\ LOUIS G. LIPPMAN, WASHINGTON STATE COLLEGE
}

Two groups of 22 Ss each were presented an 8-cluster list at a 4-sec. rate for 40 trials. All clusters (4 nonsense syllables per cluster) were identical except for the position of the syllables within each cluster. For one group the ends of the list were demarcated by a long, visually distinctive ITI; for the other group the ends of the list were disguised. The results replicated previous findings that primacy effects are eliminated when the ends of a list are disguised. Recency effects were absent in both groups.

Lippman \& Denny (1964) found that serial position effects are eliminated when the ends of a serial list are disguised (no ITI and with extralist items preceding the first regular trial). The present experiment replicated two of their groups, but under conditions of extreme intralist similarity. Different sequences of identical syllables (clusters) were used in preference to redundant sequences of letters because, with the latter, $S$ might still discover or impart special meaning to particular letters or particular letter combinations and thereby make them distinctive. Since the elements of all clusters were identical there was also a minimum of response learning. Thus, the focus was upon S's simultaneous acquisition of a sequence and the differentiation of constituent sequences.

\section{Method}

The Ss were 44 psychology students at Michigan State University. The Ss were told that four 3-letter nonsense syllables would appear; then the same set of four syllables would appear again but in a different order, again in a different order, and so on. The Ss were instructed to anticipate all four syllables by pronouncing each syllable, as it would appear, from left to right. If unsure of any syllable in a cluster, $S$ could omit it and instead say "blank." Guessing was encouraged, and Ss followed the instructions while E read them aloud. Apparatus consisted of an MTA 100 Scholar (teaching machine) and an external timer. An endless loop was used for group $C_{\text {, fanfolded paper }}$ for group $E$. The aperture was $11 / 16$ by $2-1 / 4$ in.

The list consisted of eight clusters of the same four nonsense syllables: MEP KOJ XIG ZUR; ZUR XIG MEP KOJ; XIG ZUR KOJ MEP; and so one. The four syllables of a cluster were presented horizontally in the aperture, each separated by a space of one letter width. Syllables were grouped into clusters according to the following restrictions: (a) a syllable appeared no more than twice in the same position; (b) the sequence of syllables within a cluster did not correspond to any sequence of syllables across clusters; (c) a sequence of two syllables appeared only once in those same positions; and (d) the sequence within a cluster was never reversed in another cluster. There were three additional permutations of these four syllables for use with group E. The clusters were presented in the same order on each trial for all Ss.

All clusters were presented at a $4-\mathrm{sec}$, rate. For group C, the ITI was 12 sec., consisting of two 4-sec. blank spaces and one 4-sec. presentation of three asterisks. For group E, there was no ITI, and three extralist clusters preceded the first cluster of the list on the first trial only. All Ss received 40 trials. Results and Discussion

Figure 1 presents the mean number of clusters correctly anticipated (all four syllables correctly anticipated as to position within cluster) over the eight serial positions. Analysis of variance of these data yielded a significant groups' effect $(F=11.34$, $\mathrm{df}=1 / 42, \mathrm{p}<.01$ ), indicating that group $\mathrm{C}$ correctly anticipated more clusters than group E. If all Ss anticipated each cluster with random permutations of all four syllables on every trial, a mean of 0.33 clusters would be correctly anticipated per trial by chance (perfect would be 8.00 ). Group $C$ did not consistently exceed chance until trial 16; group $\mathrm{E}$

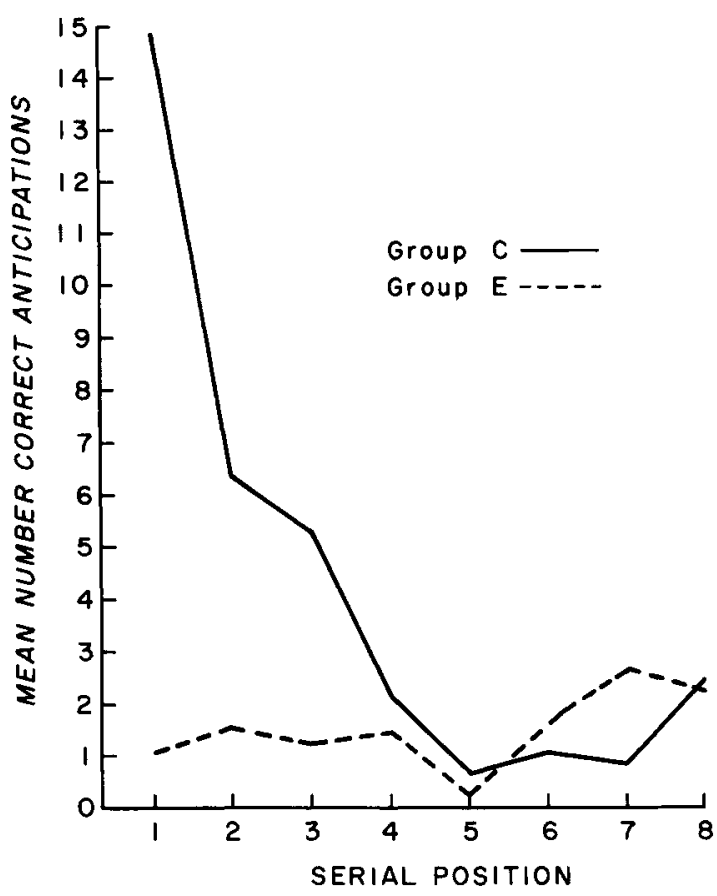

Fig. 1. Serial position curve in terms of the mean number of complete clusters correctly anticipated over all 40 trials. 
did not consistently exceed this level until trial 33 . In fact, group $\mathrm{C}$ had no more than 25 percent correctly anticipated clusters at the end of 40 trials. Learning was extremely slow in both groups.

There was a significant position effect for $\mathrm{C}$ and $\mathrm{E}$ combined $(F=14.45, \mathrm{df}=7 / 294, \mathrm{p}<.01)$. A significant position by groups interaction also obtained, indicating that the serial position curves for $\mathrm{C}$ and $\mathrm{E}$ differed in shape $(F=16.67, \mathrm{df}=7 / 294, \mathrm{p}<.01)$. Multiple comparisons by the Newman-Keuls method indicated that cluster 1 in group $C$ was correctly anticipated significantly more often than clusters 2 and 3 in group $C$ $(p<.01)$; these three clusters were in turn correctly anticipated more often than the remaining clusters $(p<.01)$. All other comparisons, involving clusters 4-8 in group $C$ and clusters $1-8$ in group $E$, were not significant. That is, group $\mathrm{E}$ did not yield the typical bow-shaped serial position curve but approximated a horizontal line. When plotted in terms of the number of correctly positioned syllables in a cluster instead of complete correct clusters, the resulting curve for group $\mathrm{E}$ was even smoother and more nearly flat (no figure included). The present results support our contention that serial position effects, conventionally plotted, are eliminated when the ends of a list are disguised. And the relative absence of peaks and valleys in the serial position curve of group E suggests that idiosyncratic item effects, which were clearly present in our 1964 study, can be reduced when intralist similarity is high.

But idiosyncratic item effects were not entirely eliminated. Group $E$ tended to show better learning of clusters 6-8 (peak at 7) than all other clusters (see Fig. 1). Of the $18 \mathrm{Ss}$ in group $\mathrm{E}$ who correctly anticipated at least one complete cluster, 11 anticipated cluster 6,7 , or 8 most frequently. Comparing this proportion to chance ( $3 / 8$ of 18 ) yielded a significant chi square, $x^{2}=4.29, \mathrm{df}=1,1<.05$. Such a result supports Murdock's contention (1960) that a list having identical distinctiveness is impossible to achieve.

Despite the bias in favor of clusters 6,7 , and 8 , per the data of group $E$, there was no evidence of a recency effect in group C. Rather, the high distinctiveness of the first cluster appeared to wash out any idiosyncratic effect from clusters 6,7 , or 8 . This was equally true when the data were scored in terms of the mean number of syllables correctly positioned within a cluster. Scored this way, cluster 5 (middle of the list) was compared with cluster 8 (end of the list). The within-group comparisons between clusters 5 and 8 for both groups $\mathrm{C}$ and $\mathrm{E}$, and the betweengroup comparisons for clusters 5 and 8 yielded nonsignificant t-test differences (all p's $>.10$ ). That is, there was no evidence of a recency effect in either group C or group E.

According to Lippman \& Denny $(1964,1966)$ the acquisition of items at the beginning of the list is facilitated by the primacy cue, while the acquisition of the end is facilitated by the recency cue. Both sets of cues are assumed to be mediated by a repeated presentation of the items in a constant order at a constant rate. The primacy cue, enhanced by the consistent position of the ITI, is associated with the first 3 or 4 items in the list and begins to function on the very first trial. On the other hand, recency is posited as a temporal or sequential cue which takes several trials to establish (e.g., about nine per Lippman and Denny). Presumably, this cue develops because a constant period of time is required to complete the list: $S$ learns when the list is about to end, and thus this point in time becomes an additional cue for learning items near the end of the list. The fact that group $\mathrm{C}$ exhibited a marked primacy effect and simultaneously failed to show any recency effect, indicates that primacy and recency effects can be considered separate processes, as posited above. In the present experiment $S$ was required to learn the eight different sequences of each cluster. These eight sequences were in addition to the sequence in which the clusters appeared. Presumably, having to learn the eight additional sequences interfered with S's ability to learn the sequential cue for the list as a whole (recency) but did not prevent the operation of primacy which did not need to be learned as a cue.

\section{References}

Lippman, L. G., \& Denny, M. R. Serial position effect as a function of intertrial interval. J. verbal Learn. verbal Behav., 1964, 3, 496-501.

Lippman, L. G., \& Denny, M. R. Comment on the role of the intertrial interval in serial learning: A clarification. Psychon. Sci., $1966,4,234$.

Murdock, B. B., Jr. The distinctiveness of stimuli. Psychol. Rev... $1960,67,16-31$ 